\title{
Characterizing Fluid and Kinetic Instabilities using Field-Particle Correlations on Single-Point Time Series
}

\author{
Kristopher G. Klein ${ }^{1}$, 国 \\ Department of Climate and Space Sciences and Engineering, University of Michigan, Ann Arbor, \\ MI 48109, USA
}

\begin{abstract}
A recently proposed technique correlating electric fields and particle velocity distributions is applied to single-point time series extracted from linearly unstable, electrostatic numerical simulations. The form of the correlation, which measures the transfer of phase-space energy density between the electric field and plasma distributions and had previously been applied to damped electrostatic systems, is modified to include the effects of drifting equilibrium distributions of the type that drive counter-streaming and bump-on-tail instabilities. By using single-point time series, the correlation is ideal for diagnosing dynamics in systems where access to integrated quantities, such as energy, is observationally infeasible. The velocity-space structure of the field-particle correlation is shown to characterize the underlying physical mechanisms driving unstable systems. The use of this correlation in simple systems will assist in its eventual application to turbulent, magnetized plasmas, with the ultimate goal of characterizing the nature of mechanisms that damp turbulent fluctuations in the solar wind.
\end{abstract}

\section{INTRODUCTION}

A significant goal of plasma physics research is the characterization of mass, momentum, and energy transport in a wide variety of complex systems. In particular, the question of what mechanisms mediate the transfer of energy between turbulent fields and distributions of plasma particles, leading to the eventual damping and dissipation of turbulence, is open. One system which displays turbulent behavior is the solar wind, a hot, diffuse emanation from the Sun's surface that fills the heliosphere. While lacking the precise control over conditions afforded in a laboratory setting, the large volume of in situ measurements of the solar wind over the last half century has led to the accrual of observations of turbulence with a wide variety of plasma parameters. Such measurements have proven useful to the study of phenomena in magnetized turbulence $\underline{\underline{1}}$

A limitation of these in situ observations is that they are taken at a single-point in space at a given time $\stackrel{2}{2}$ This raises at least two significant complications; one must disentangle the dynamics associated with spatial and temporal variation, and track the evolution of spatially integrated quantities, such as the energy content of a field or distribution of charged particles, given only single-point measurements. The first of these complications is addressed by invoking Taylor's Hypothesis, $\frac{3}{-}$ the conjecture that for single-point measurements of sufficiently

a) Electronic mail: kriskl@umich.edu fast flows, the time evolution is essentially frozen and the measurement traces out the spatial structure of the turbulence; a review of the application of Taylor's Hypothesis to solar wind observations can be found in Klein et al 2014.

To address the second complication of inaccessible spatially integrated quantities, one may consider the dynamics of the phase-space energy density rather than the total energy. A technique has been recently proposed to measure the local-in-phase-space energy transfer between fields and plasma distributions using single-point time series of simple plasma

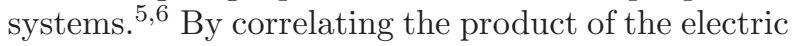
field and velocity derivative of the particle distribution measured at a single point in space, the velocity structure of the transfer of energy between fields and particles is obtained. By averaging this correlation over a selected time interval, the oscillatory energy transfer between the fields and particles is removed, leaving only the secular energy transfer. The mechanisms responsible for this energy transfer can be identified by the velocity space structure of the field-particle correlation. Initial work applied this correlation to systems that damp via the Landau resonance ${ }^{\underline{7}}$ Here, we consider the transfer of energy in linearly unstable systems, and show that fieldparticle correlations are able to identify the presence of both fluid and kinetic instabilities in such systems.

The unstable systems under consideration are reviewed in Section III In Section III, we present the nonlinear numerical code employed in this work, VP, as well as the three simulations under consideration. In Section IV the field-particle correlation is presented and applied to the three simulations. Anal- 
ysis and discussion related to the correlations are found in Section V Application of field-particle correlations to simple, homogeneous, linearly unstable systems enables the construction of signatures of basic energy transfer mechanisms. Combined with signatures for all relevant energy transfer mechanisms, such correlations may be usefully employed to diagnose the behavior of more complex systems.

\section{LINEARLY UNSTABLE SYSTEMS}

The 1D1V electrostatic systems of interest in this work are governed by the Vlasov and Poisson equations

$$
\frac{\partial f_{s}}{\partial t}+v \frac{\partial f_{s}}{\partial x}-\frac{q_{s}}{m_{s}} \frac{\partial \phi}{\partial x} \frac{\partial f_{s}}{\partial v}=0
$$

and

$$
\frac{\partial^{2} \phi}{\partial x^{2}}=-4 \pi \sum_{s} q_{s} \int_{-\infty}^{\infty} d v f_{s}
$$

which evolve the distribution of species $s, f_{s}(x, v, t)$ and the electric field $E(x, t)=-\partial \phi(x, t) / \partial x$. Recent work has applied field-particle correlations to electrostatic stable systems as a means of extracting the velocity dependent signature of Landau damp-

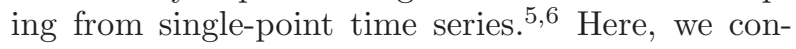
sider electrostatic systems unstable to either fluid or kinetic instabilities, and characterize the signature of the associated growth using field-particle correlations. For fluid instabilities, the behavior of the plasma depends on the bulk parameters of the system and the energy transfer is not organized by characteristic velocities such as the resonant wave phase speed; energy transfer for kinetic instabilities depends on such characteristic velocities, as the electrostatic field acts to exchange particles of higher and lower kinetic energies near the field's resonant velocity. For both types of instabilities, one or more of the distributions lose energy, while the fields and other distributions gain energy, resulting in an inverse damping. General discussion of plasma instabilities, as well as particular treatments of the electron drift instabilities of interest in this work, can be found in many plasma textbooks. $\stackrel{-10}{-10}$

To highlight the distinct velocity space structure of energy transfer in unstable systems, we consider three cases, each with distinct sets of parameters for one population of ions and two populations of electrons. The equilibrium distributions for the three
TABLE I. Electron Bulk Parameters

\begin{tabular}{|c|c|c|c|c|}
\hline & $n_{e 1} / n_{i}$ & $v_{d 1} / v_{t e}$ & $n_{e 2} / n_{i}$ & $v_{d 2} / v_{t e}$ \\
\hline Case 1 & 0.5 & 0.75 & 0.5 & -0.75 \\
Case 2 & 0.5 & 1.75 & 0.5 & -1.75 \\
Case 3 & 0.9 & 0.00 & 0.1 & 4.25 \\
\hline
\end{tabular}

populations take the Maxwellian form

$$
F_{s 0}(v)=\frac{n_{s j}}{\sqrt{2 \pi}} \exp \left[\frac{-\left(v-v_{d s j}\right)^{2}}{2 v_{t s}^{2}}\right]
$$

where $v_{d s j}$ is the population's drift velocity. The linear normal mode behavior for such equilibria is governed by solutions of the dispersion relation

$\underline{\underline{D}}(\omega, k)=k^{2} \lambda_{D e}^{2}+\sum_{j}\left(\frac{q_{s}}{q_{e}}\right)^{2} \frac{n_{s j}}{n_{i}} \frac{T_{e}}{T_{s}}\left[1+\xi_{s j} Z_{0}\left(\xi_{s j}\right)\right]$

where $\underline{\underline{D}}$ is a function of wavenumber $k$ and complex frequency $(\omega, \gamma), q_{s}$ is the species charge, $Z_{0}$ is the plasma dispersion function ${ }^{11}$ with argument $\xi_{s j}=$ $\left(\omega / \omega_{p e} k \lambda_{D e}\right)\left(\sqrt{T_{e} m_{s} / 2 T_{s} m_{e}}\right)-v_{d s j} / v_{t s}$, with the sum taken over the three plasma populations $j$. The electron plasma frequency $\omega_{p e} \equiv \sqrt{4 \pi \sum_{j} n_{e j} q^{2} / m_{e}}$ and Debye length $\lambda_{D e}=\sqrt{T_{e} / 4 \pi \sum_{j} n_{e j} q^{2}}$, both defined using the total electron density, normalize the time and length scales of our system. Values for the normalized density of electron population $j, n_{e j} / n_{i}$, and bulk velocity, normalized by the electron thermal velocity $v_{t e}=\sqrt{T_{e} / m_{e}}$, are given in Table【. For all three cases, the electron populations have equal temperatures; the ions are singly ionized, and initialized with $T_{i}=T_{e}, m_{i}=100 m_{e}$, and $v_{d i}=0$. Case 1 is stable to the effects of the counter-streaming electrons, while the increase in $\left|v_{d e j}\right|$ for case 2 yields the classic counter-streaming instability. Case 3 is unstable to the bump-on-tail instability. Cases 2 and 3 serve as examples of fluid and kinetic instabilities respectively.

In Fig 1] solutions to Eqn 4 for the three cases are presented. In panel a, the complex frequency solutions $(\omega, \gamma) / \omega_{p e}$ are given for fixed $k \lambda_{D e}=0.2$. For case 1 (black circles) all modes are shown to be damped. For case 2 (red triangles), the increase in $\left|v_{d e j}\right|$ leaves the frequency and damping rate of the Langmuir modes, the modes with $|\omega| \approx \omega_{\text {pe }}$, largely unaffected. The pair of least damped acoustic modes from case 1 are now both unstable, having $\omega=0$ 

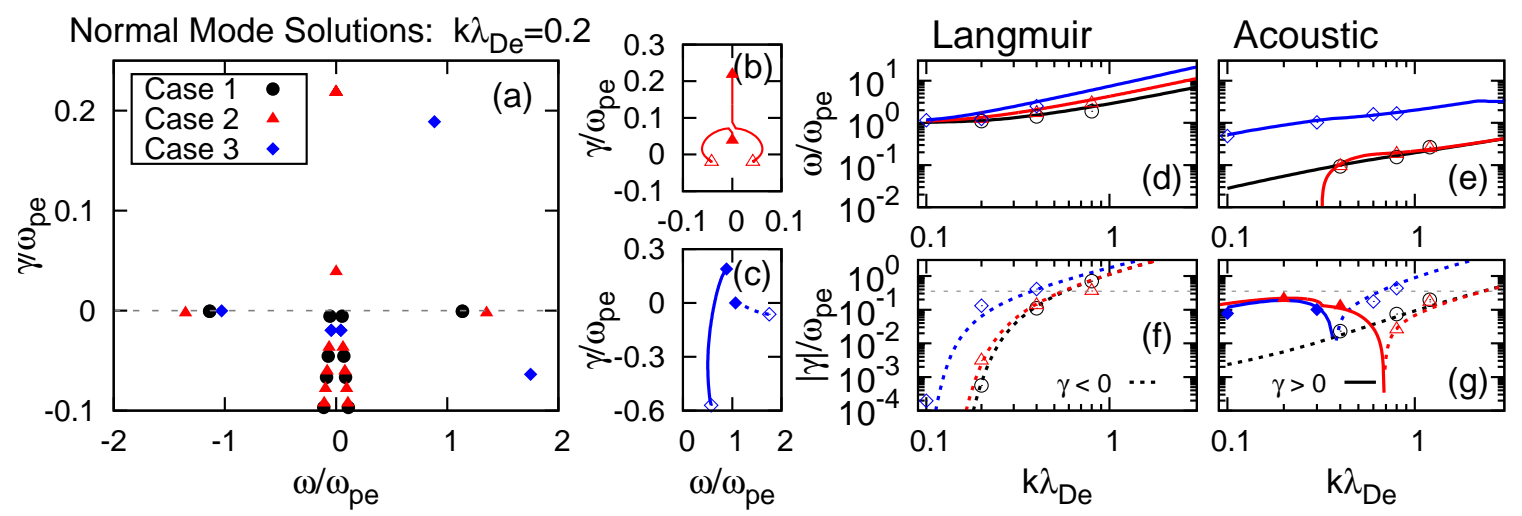

FIG. 1. Normal mode properties for the three cases examined in this work. The complex frequency solutions to Eqn 4 for $k \lambda_{D e}=0.2$ and the parameters of cases 1, 2, and 3 (black, red, and blue) are shown in panel a. Parametric paths for normal mode solutions from $\left|v_{d e j}\right|=0\left(v_{d e 2}=0\right)$ to $\left|v_{d e j}\right|=1.75\left(v_{d e 2}=4.25\right)$ are plotted in panel b (c), indicating the connection between stable and unstable acoustic modes. Frequency dispersion curves $\omega\left(k \lambda_{D e}\right) / \omega_{p e}$ for the least damped and/or fastest growing Langmuir and acoustic modes are illustrated in panels d and e. Damping rates $\gamma\left(k \lambda_{D e}\right) / \omega_{p e}$ for the same modes are plotted in panels $\mathrm{f}$ and $\mathrm{g}$, with solid (dashed) lines indicating growth (damping). Frequency estimates extracted from linear and small amplitude nonlinear VP simulations are shown as points in panels $\mathrm{d}-\mathrm{g}$.

and two distinct growth rates, $\gamma>0$. The parametric path from stability $\left(\left|v_{d e j}\right|=0.0\right.$, open triangles $)$ to instability $\left(\left|v_{d e j}\right|=1.75\right.$, filled triangles $)$ for these two modes is illustrated as a function of complex frequency in panel b. For case 3, the $\omega<0$ Langmuir mode and the least damped acoustic modes are negligibly affected by the bump distribution. By parametric variation of $v_{d e 2}$ from 0.0 (open diamonds in panel c) to 4.25 (filled diamonds), it is observed that the growing mode for the bump-on-tail instability arises from an acoustic mode which is strongly damped in the stable regime (solid line), while the $\omega>0$ Langmuir mode (dashed line) becomes heavily damped. The dispersion relations for the least damped and/or fastest growing Langmuir (acoustic) modes as a function of $k \lambda_{D e}$ for the three cases are presented in panels $\mathrm{d}$ and $\mathrm{f}$ ( $\mathrm{e}$ and $\mathrm{g}$ ) illustrating the wavelengths for which linear instabilities arise for cases 2 and 3 (solid lines in panel $\mathrm{g}$ ).

\section{NUMERICAL SIMULATIONS}

To evaluate the time evolution of these systems, we have extended the Vlasov-Poisson solver VP ${ }^{6}$ to allow for the inclusion of an arbitrary number of drifting plasma populations. VP solves the nonlinear Vlasov-Poison system using second-order finite differencing for spatial and velocity derivatives and a third-order Adams-Bashforth scheme in time. As a test of this extension, we compare numerical solutions of Eqn. 4 for the three cases described in section II to frequencies and damping rates extracted from time traces of the electrostatic field energy from linear and small-amplitude nonlinear VP simulations for a range of wavevectors, given as points in Fig. 1] Agreement between the dispersion relation and VP are close as long as the mode of interest is not heavily damped and unstable modes supported by the system do not grow too quickly with respect to the damped modes.

For the evaluation of the field-particle correlation, we perform three nonlinear simulations corresponding to the three cases from section II] For these simulations, we add a sinusoidal perturbation to the ion's equilibrium distribution of the form $0.1 F_{i 0}(x, v) \sin \left(k_{0} x\right)$ with $k_{0} \lambda_{d e}=0.2 .256$ (128) points in velocity (coördinate) space are resolved over the interval $v / v_{t s} \in[-8,8]\left(x / \lambda_{D e} \in\right.$ $[-5 \pi, 5 \pi])$. Each simulation is run for longer than $t=40 \omega_{p e}^{-1}$ and the total energy in the system

$$
W_{\text {total }}=\int d x \frac{E^{2}}{8 \pi}+\sum_{s} \int d x \int d v \frac{m_{s} v^{2}}{2} f_{s}
$$

is conserved to better than a few tenths of a percent. Changes in the electrostatic energy $W_{\phi}=$ $\int d x E^{2} / 8 \pi$ as well as energy in the three plasma populations, $W_{j}=\int d x \int d v m_{j} v^{2} f_{j} / 2$, from their 

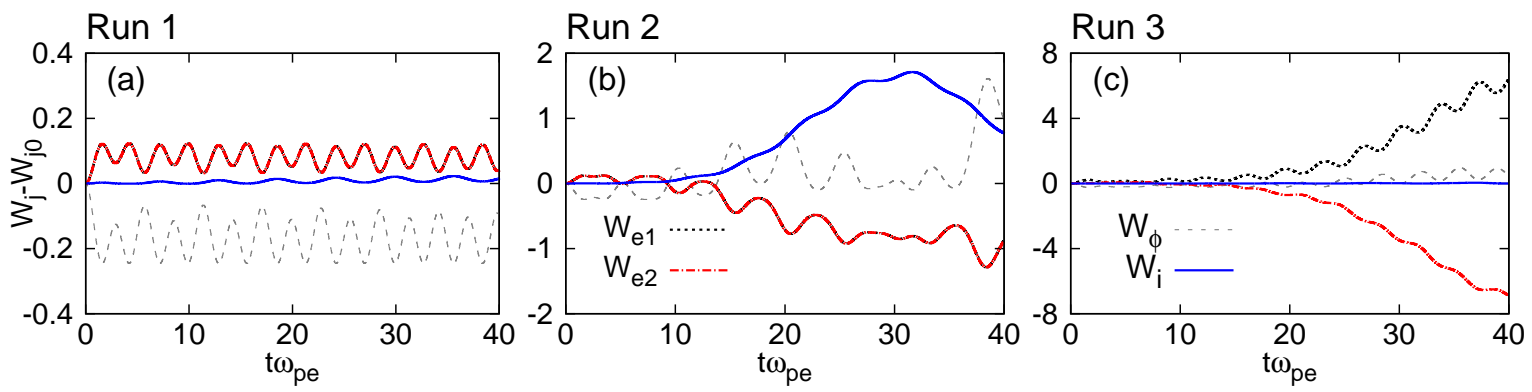

FIG. 2. The change in energy, $W_{j}(t)-W_{j}(t=0)$, for the two populations of electrons, the ions, and the electrostatic field (black, red, blue, and grey lines), for the three nonlinear simulations, normalized by $T_{e}$.

initial values are shown in Fig. 2. with energy normalized by $T_{e}$. In run 1, most of the energy damped from the electric field is equally partitioned between the two electron populations, with little energy transferred to the ions. For run 2, an instability is clearly triggered, with significant losses of energy from the electrons and both $W_{\phi}$ and $W_{i}$ growing from their initial values. A more virulent instability is triggered in run 3, with the bump electron population losing a significant fraction of its energy to the core electron population, with little energy transferred to the ions.

\section{FIELD-PARTICLE CORRELATIONS}

While tracking the change in energy is sufficient to identify instabilities in systems with complete knowledge of spatial and velocity structure, we seek the signature of unstable behavior given limited, singlepoint measurements of a system of the type available to spacecraft in the solar wind. The application of field-particle correlations to such measurements allows for a local observation of secular energy transfer.

We define the field-particle correlation for a discrete set of measurements of $f_{s}(x, v, t)$ and $E(x, t)$ with timestep $d t$, taken at a single point $x=x_{0}$ as

$C_{E}\left(x_{0}, v, t_{i}, N\right)=-\frac{1}{N} \sum_{j=i}^{i+N} \frac{q_{s} v^{2}}{2} \frac{\partial f_{s}\left(x_{0}, v, t_{j}\right)}{\partial v} E\left(x_{0}, t_{j}\right)$

The correlation averages the field-particle interaction term in the Vlasov equation, the third term in Eqn. 1, over a time interval of length $\tau=N d t$. As the ballistic term, the second in Eqn. 1 does not lead to net energy transfer, $\stackrel{6}{=}$ the product in $C_{E}$ represents the energy density transferred at one point in phase

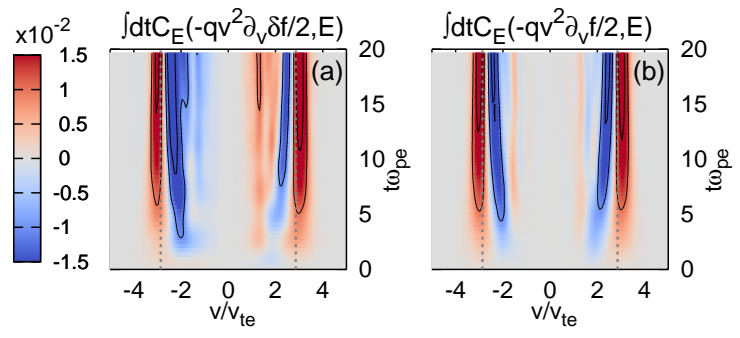

FIG. 3. The accumulated change in the electron phasespace energy density for damped, counter-propagating Langmuir waves calculated using the perturbed electron distribution in panel a, Eqn. 2 from Klein \& Howes $2016^{\frac{5}{5}}$ and the total electron distribution in panel b, Eqn. 6. The resonant velocities for the system are indicated by dashed grey lines.

space between the electric field and velocity distribution. By averaging over a selected time interval $\tau$, oscillatory energy transfer between $E$ and $f_{s}$ is removed, leaving only the secular energy transfer. To track the accumulated change of the phase-space energy density, we integrate the correlation over time, defining $\Delta w_{s}(x, v, t, N) \equiv \int_{0}^{t} d t^{\prime} C_{E}\left(x, v, t^{\prime}, N\right)$.

Unlike Eqn. 2 in Klein \& Howes $2016, \frac{5}{\underline{5}}$ we include the full distribution function in our definition of $C_{E}$, rather then only the perturbed component $\delta f_{s}$. Previous studies had focused on particular cases where the equalibria $F_{s 0}$ were even with respect to $v=0$, ensuring that they would not contribute to net energy transfer. For the cases under consideration in this work, the equilibrium electron distributions have odd components and therefore may contribute to a secular transfer of energy between the fields and distributions. To show that the two forms of the correlation obtain similar results when $F_{s 0}$ is even, we apply both correlations to single-point field and distribution data from a Landau damped counter- 
propagating Langmuir wave simulation, case 1 in Klein \& Howes 2016, and plot $\Delta w_{e}$ at $x=0$ with $\tau \omega_{p e}=6.28$ in Fig. 3. We see that both correlations produce qualitatively similar structure in the accumulated phase-space energy density, especially in regards to production of a plateau surrounding the resonant velocities of the system, $\left|v_{\text {res }}\right|=2.86 v_{t e}$, which serves as the key velocity-space signature of Landau damping.

With our correlation defined, we next select an appropriate correlation interval $\tau$ for the three simulations. By averaging over a particular interval, we remove the transfer of energy between the fields and particles which oscillates with frequency of the order $\omega \sim 2 \pi / \tau$, leaving the secular, or non-oscillatory, component. Plotted in Fig. 4 are velocity integrated correlations, $\int d v C_{E}(x, v, t, \tau)$, between $E$ and the three plasma populations for a range of correlation lengths $\tau \omega_{p e} \in[0,30]$ at a single spatial location $x=0$.

There is significant oscillatory transfer for small $\tau$ correlations for run 1, panels a-c of Fig. 4. This oscillatory transfer is reduced for longer intervals, with nearly all of the oscillations removed for $\tau \omega_{p e}=5.64$, a correlation length corresponding to the period of Langmuir waves supported by the system with frequency $\omega=1.11 \omega_{p e}$. This $\tau$ leaves the velocity integrated correlations for all three distribution functions nearly monotonic, while slightly longer correlations reintroduce some oscillatory behavior. As the Langmuir wave is the least damped, finite frequency linear mode supported by this system, correlating over its period is physically justified. For run 2, panels d-f, correlating over the interval $\tau \omega_{p e}=5.15$, which corresponds to the Langmuir wave frequency $\omega=1.21 \omega_{p e}$, removes significant oscillatory energy transfer. Averaging over the time scale associated with the least damped modes, rather than the unstable modes, is motivated by the fact that the unstable modes of this system have zero frequency; see Fig [1. For run 3, we see that there does not exist a single value of $\tau$ for which the oscillatory energy transfer is completely removed. This due to the fact that the system supports both a weakly damped Langmuir wave as well as a finite-frequency unstable acoustic mode. Correlating over the Langmuir period retains some of the acoustic oscillations, while correlating over the acoustic period retains some of the Langmuir oscillations. We choose $\tau \omega_{p e}=7.11$, corresponding to the period of the growing acoustic mode with $\omega=0.88 \omega_{p e}$ and acknowledge that some oscillatory contributions from Langmuir waves will persist.

\section{VELOCITY-SPACE STRUCTURE OF ENERGY TRANSFER}

With an appropriate interval $\tau$ selected, we calculate the velocity dependent field-particle correlation for the three simulations. By retaining the velocity dependence, we are able to address the question of where the energy transfer occurs in phase space, and use the structure of this energy transfer to characterize the nature of the underlying instability.

For the stable, counter-streaming electron case, run 1 , the phase-space energy transfer obtained from the field-particle correlation is a fairly regular function of velocity. At a given point in coördinate space, for example $x=0 \lambda_{D e}$ shown in the first row of Fig. 5. one of the electron populations gains energy from, while the other population loses energy to, the electric field. The energy transfer to the ions from the electric field is an odd function of velocity, meaning that when the correlation is integrated over $v$, there is no net energy transfer to the ions. There is no evidence for the dependence of the phase-space energy transfer on drift velocities (black vertical dashed lines) or the resonant velocities of either the Langmuir (green lines) or acoustic (magenta) modes for any of the three populations.

Increasing the speed of the counter-streaming electrons for run 2, shown in panels e-h of Fig. 5, the structure of the energy transfer is altered. The sign of the field-particle correlation changes at $v_{d e j}$ due to a change in sign of $\partial f_{e j} / \partial v$. Unlike for case 1 , this change in sign occurs for electrons which exchange significant energy with the electric field. For the ions, a small, even component in the field-particle correlation arises, yielding a net transfer of energy from the fields to the ions, as seen in panel $\mathrm{h}$. This transfer of energy to the ions, which does not depend on either the Langmuir or acoustic resonant velocities, serves as a phase-space signature for the fluid instability that arises for this system.

For the bump-on-tail instability, case 3 shown in panels i-l of Fig. 5, the velocity-space structure of the field-particle correlation is significantly different. As we have correlated over the unstable acoustic period, the oscillatory structure from the weakly damped Langmuir mode is evident. We also see in the bump electron population that the energy transfer changes sign across the acoustic resonance and at later time across the Langmuir resonance. This resonant structure is the signature of the transfer of energy from the bump population to the core electron population as mediated by the electric field. We note that the resonant structure is maintained for other 

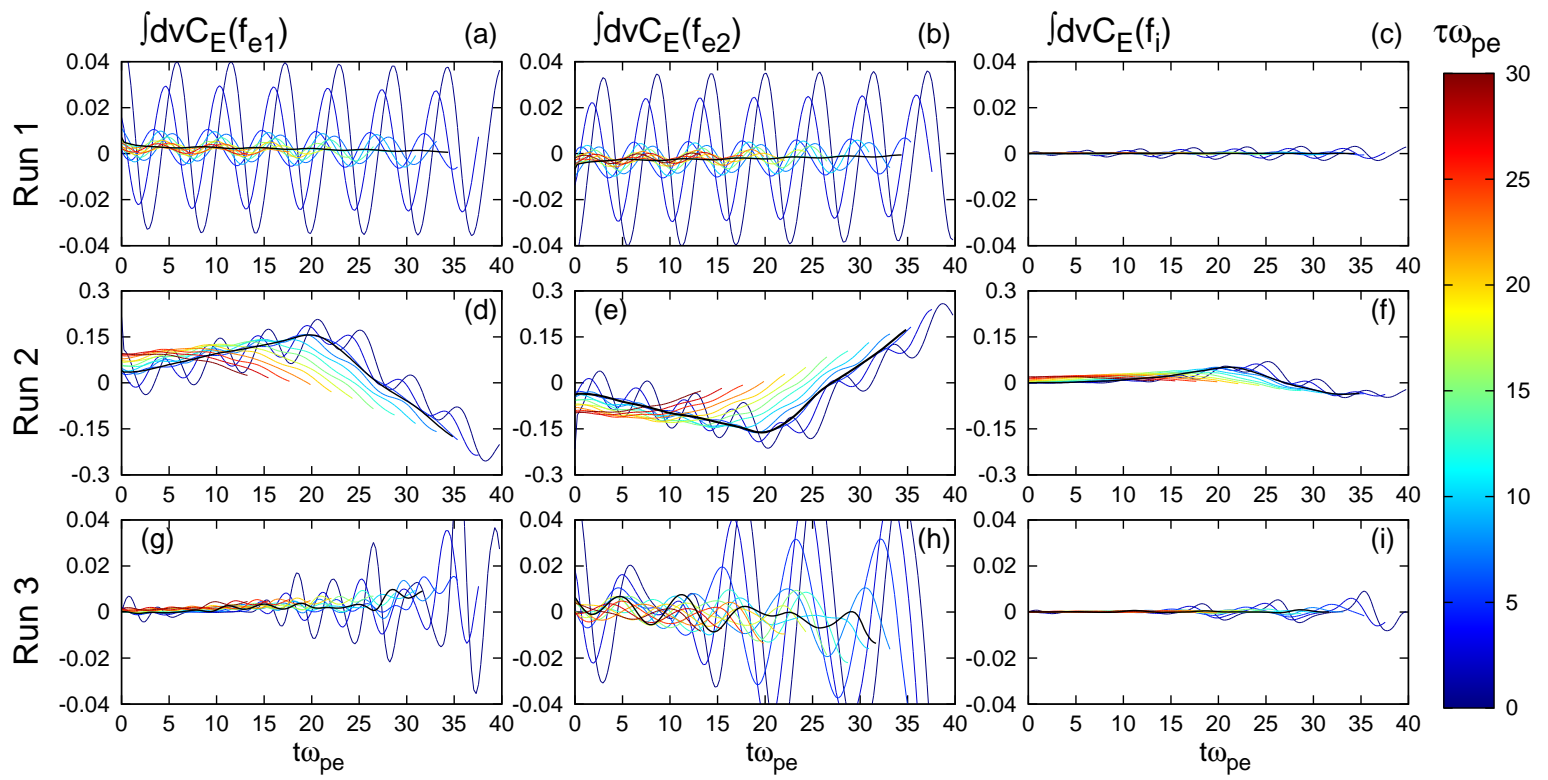

5

FIG. 4. The velocity integrated field-particle correlation $C_{E}\left(f_{s}\right)$ at $x=0$ for a range of correlation intervals $\tau \in[0,30]$, differentiated in color. Each row represents the three simulations under consideration, while the columns present the correlation with $f_{e 1}, f_{e 2}$ and $f_{i}$. The black line highlights the $\tau$ selected for Fig 5 .

choices of $\tau$, including an interval corresponding to the Langmuir wave period, not shown. The velocityintegrated correlation confirms that the core population has a net gain of energy, and as expected for this instability, we see that the electrons that receive the energy are near the resonant velocities. This explicit dependence of the phase-space energy transfer on resonant coupling between the fields and particles serves as a distinct signature of kinetic instabilities when compared to the fluid instability in run 2 .

The field-particle correlations presented in Fig. 5 were calculated at a single point in coördinate space. An obvious question arises as to how the correlation and the associated phase-space energy transfer change as a function of position within the simulation. To asses this question, we calculate $C_{E}$ at five other points within the simulation and present the velocity integrated accumulated change of the phase-space energy density, $\int d v \Delta w_{s}$, in Fig. 6. For the stable case, run 1 , we see that the energy transfer to or from the two electron populations changes sign and amplitude as a function of the position in the simulation, with the transfer passing through zero at the two nodes of the initial standing wave pattern at $\pm 7.9 \lambda_{D e}$. The correlation with the ions maintains an even velocity space structure such that the ions continue to neither lose nor gain net energy from the electric field regardless of spatial position. For the unstable counter-streaming electrons, run 2, the same pattern of shifting sign and amplitude for the energy transfer to and from the electron holds. The ions gain energy from the electric field regardless of which electron population is gaining or losing energy. The amplitude of the ion energy gain changes with the amplitude of the electron field-particle correlation, going to zero at the nodes and having its largest value at the anti-nodes. This phase space structure serves as a distinct signature of growing fluid instabilities. For the bump-on-tail instability, run 3 , there is no regular spatial variation in the transfer of energy between the beam and core electron populations; the core gains energy at the expense of the beam, with the energy density accumulating at nearly the same rate regardless of spatial position, as expected for a kinetic instability.

\section{CONCLUSION}

Field-particle correlations of the form defined in Eqn. 6, modified from Klein \& Howes $2016^{\underline{5}}$ to account for drifting equilibrium distributions, are applied to a set of simulations where fluid and kinetic instabilities are present. The structure of the result- 

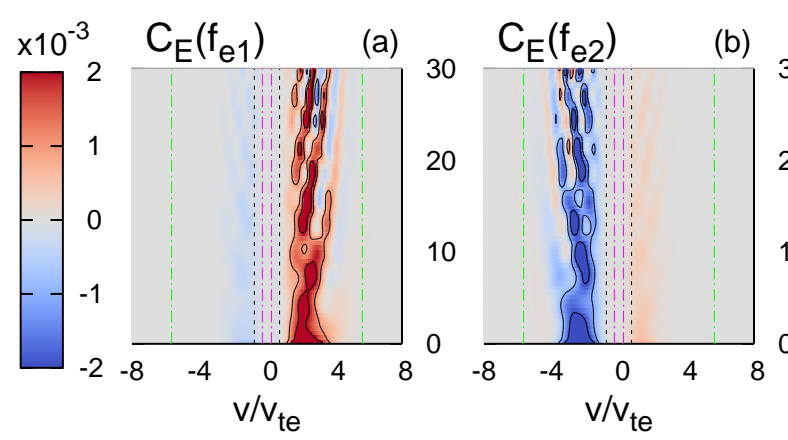

b)

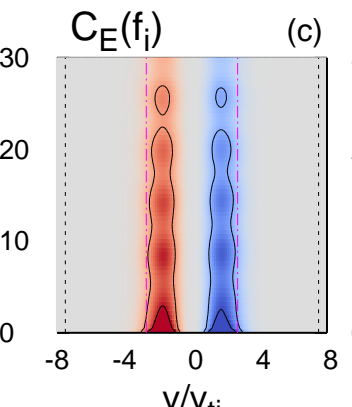

(c)
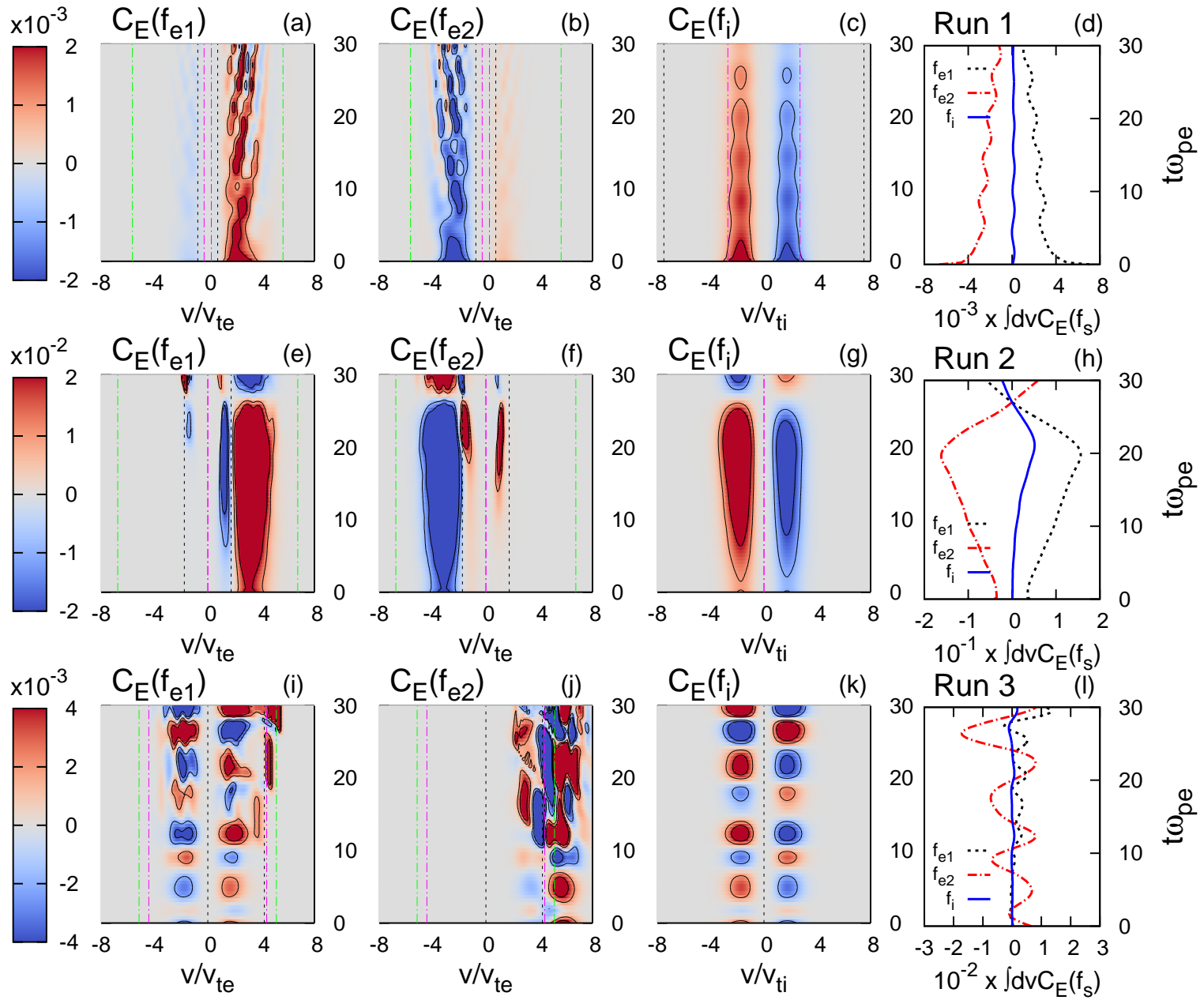

FIG. 5. The velocity dependent field-particle correlation $C_{E}\left(f_{s}\right)$ at $x=0$ for runs 1 (panels a-c), 2 (panels e-g), and 3 (panels i-k) as well as the velocity integrated correlation for $f_{e 1}, f_{e 2}$, and $f_{i}$ (black, red, and blue lines in panels $\mathrm{d}$, h, and 1), for $\tau \omega_{p e}=5.64$ (run 1), 5.15 (run 2), and 7.11 (run 3). The vertical dashed black lines indicate the electron drift velocities $v_{d 1} / v_{t e}$ and $v_{d 2} / v_{t e}$, while the green and magenta vertical lines give the resonant velocities for the least damped and/or fasted growing Langmuir and acoustic waves respectively.

ing correlations, which can be interpreted as the secular phase-space energy density transferred between the fields and distributions, can be used to identify the presence of instabilities as well as to characterize the mechanisms driving the unstable growth. The form of the correlation allows for such characterizations to be made from observations at a single point, or a few points, in coördinate space, as opposed to requiring knowledge of spatially integrated quantities typically not accessible to experimental measurements.

We consider simplified 1D-1V electrostatic systems in an attempt to characterize field-particle correlations as measurements of secular energy transfer in advance of future work applying such correlations to systems of higher dimensionality, where magnetization, turbulence, and inhomogeneities may complicate the interpretation of the correlation. By determining signatures of basic plasma physics phenomena responsible for energy transfer between fields and distributions, such as Landau damping and one-dimensional instabilities, we lay the foundation for the determination of the velocity distribution signatures of more complex interactions, such as cyclotron 9 and transit time damping, 12 heating by large amplitude, stochastic fluctuations, 13 and magnetic reconnection,,$\frac{14}{}$ which have all been proposed to play a role in the dissipation of turbulent fluctu- 


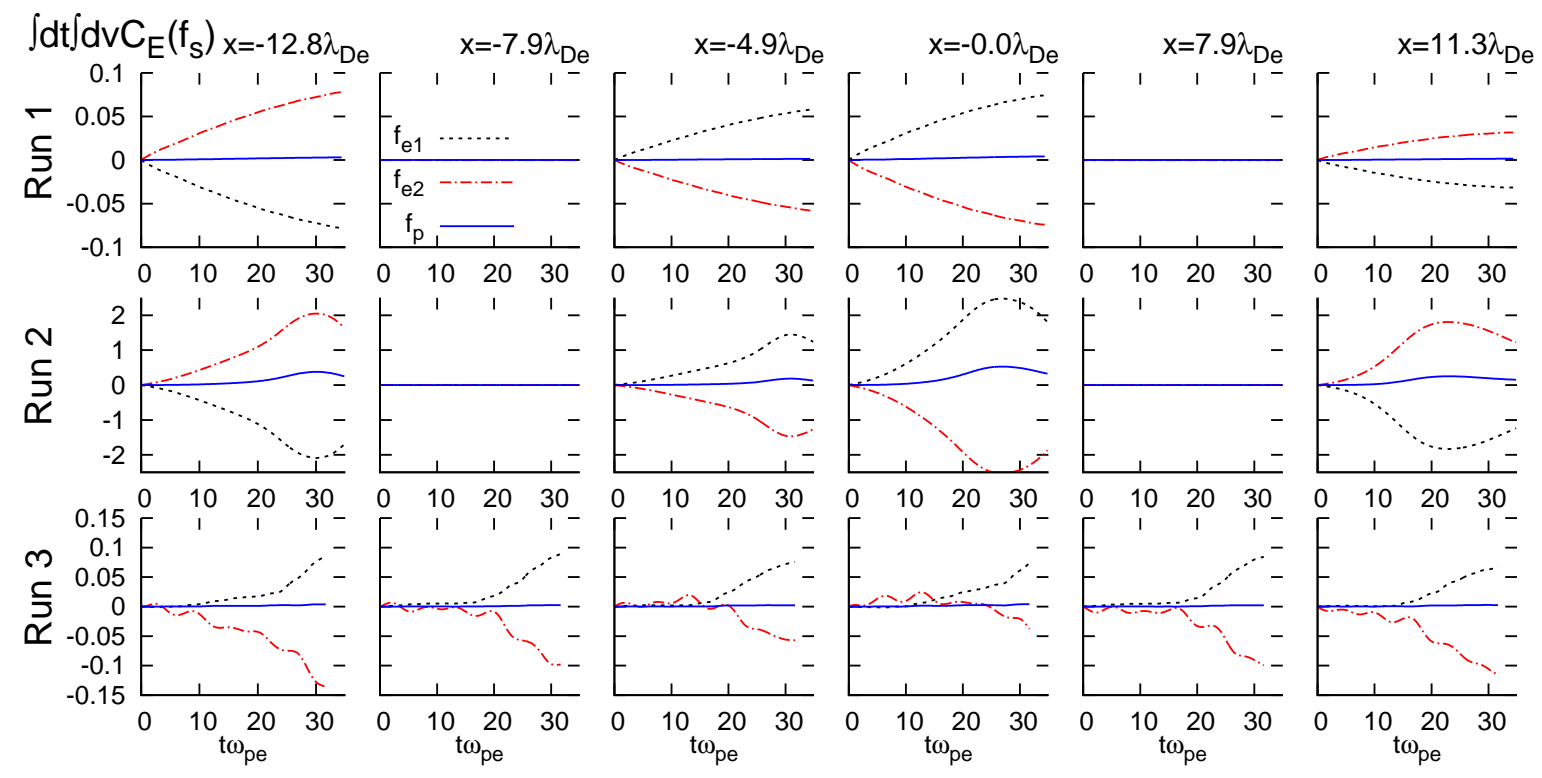

FIG. 6. The time and velocity integrated field-particle correlation $\int d t \int d v C_{E}\left(f_{s}\right)$ at six points in coördinate space, arranged by columns, with values for $f_{e 1}, f_{e 2}$, and $f_{i}$ given in black, red, and blue. The top, central, and bottom rows illustrate values for runs 1,2 , and 3 .

ations. Future work will also consider the effects of solar wind expansion, electron conduction, and other inhomogeneous mechanisms on the plasma to clearly identify the role of such energy transfer mechanisms in the solar wind. By constructing the correlation to be obtained from single-point measurements, we allow for the identification of such damping mechanisms from in situ observation of the solar wind on current and future missions including Deep Space Climate Observatory (DSCOVR), MMS, 15 and Solar Probe Plus $\underline{\underline{16}}$

\section{ACKNOWLEDGMENTS}

The author would like to thank Gregory Howes, Justin Kasper, and Jason TenBarge for insightful discussions regarding aspects of this work. This research was supported by the NASA HSR grant NNX16AM23G.

\section{REFERENCES}

${ }^{1}$ R. Bruno and V. Carbone, Living Rev. Solar Phys. 2, 4 (2005).

${ }^{2}$ Notable exceptions to the single-point limitation are the are the Cluster $\stackrel{17}{=}$ THEMIS,,$\frac{18}{\longrightarrow}$ and Mag- netospheric Multiscale (MMS) 15 missions, which are comprised of four or five spacecraft arranged in particular geometric configurations.

${ }^{3}$ G. I. Taylor, Royal Society of London Proceedings Series A 164, 476 (1938).

${ }^{4}$ K. G. Klein, G. G. Howes, and J. M. TenBarge, Astrophys. J. Lett. 790, L20 (2014).

${ }^{5}$ K. G. Klein and G. G. Howes, Astrophys. J. Lett. 826, L30 (2016).

${ }^{6}$ G. G. Howes, K. G. Klein, and T. C. Li, J. Plasma Phys. (under review).

${ }^{7}$ L. D. Landau, J. Phys.(USSR) 10, 25 (1946), [Zh. Eksp. Teor. Fiz.16,574(1946)].

${ }^{8}$ N. A. Krall and A. W. Trivelpiece, Principles of plasma physics, McGraw-Hill, 1973.

${ }^{9}$ T. H. Stix, Waves in plasmas, American Institute of Physics, 1992.

${ }^{10}$ R. D. Hazeltine and F. L. Waelbroeck, The framework of plasma physics, Westview, 2004.

${ }^{11}$ B. D. Fried and S. D. Conte, The Plasma Dispersion Function, Academic Press, 1961.

${ }^{12}$ A. Barnes, Phys. Fluids 9, 1483 (1966).

${ }^{13}$ B. D. G. Chandran, B. Li, B. N. Rogers, E. Quataert, and K. Germaschewski, Astrophys. J. 720, 503 (2010).

${ }^{14}$ M. Yamada, R. Kulsrud, and H. Ji, Reviews of Modern Physics 82, 603 (2010).

${ }^{15}$ J. L. Burch, T. E. Moore, R. B. Torbert, and B. L. Giles, Space Science Reviews 199, 5 (2016). 
${ }^{16}$ N. J. Fox et al., Space Sci. Rev. (2015).

${ }^{17}$ C. P. Escoubet, M. Fehringer, and M. Goldstein, Annales Geophysicae 19, 1197 (2001).
${ }^{18}$ V. Angelopoulos, Space Science Reviews 141, 5 (2008). 